\title{
Cuisine et bonheur : la philosophie de Ferran Adrià
}

Paloma OTAOLA

\section{(2) OpenEdition \\ Journals}

Édition électronique

URL : http://journals.openedition.org/transtexts/627

DOI : $10.4000 /$ transtexts. 627

ISSN : 2105-2549

Éditeur

Gregory B. Lee

Référence électronique

Paloma OTAOLA, «Cuisine et bonheur : la philosophie de Ferran Adrià », Transtext(e)s Transcultures 跨 文本跨文化 [En ligne], 10 | 2015, mis en ligne le 05 août 2016, consulté le 30 avril 2019. URL : http:// journals.openedition.org/transtexts/627 ; DOI : 10.4000/transtexts.627

Ce document a été généré automatiquement le 30 avril 2019.

(c) Tous droits réservés 


\title{
Cuisine et bonheur : la philosophie de Ferran Adrià
}

\author{
Paloma OTAOLA
}

1 Depuis sa première étoile Michelin comme chef de «El Bulli » (Girona-Espagne) en 1990, Ferran Adrià (1962-) a poursuivi une carrière couronnée de succès, devenant ainsi la figure emblématique de la nouvelle cuisine et de la gastronomie espagnoles. Adrià est aujourd'hui un personnage médiatique et populaire, non seulement en Espagne mais aussi à l'étranger.

2 Ses réalisations et ses créations innovantes dans le domaine de la gastronomie sont devenues populaires à travers la télévision, les catalogues, les livres, les documentaires et autres moyens de diffusion. Si d'une part, son succès international a nourri la fierté des Espagnols, d'autre part, la philosophie de Ferran Adrià sur les aliments, leur élaboration en cuisine, la gastronomie, l'art de la table, etc., a eu un grand impact culturel et social. Grâce à son travail, l'art culinaire est devenu un facteur intégrant de la culture et du savoir-vivre d'un pays et d'une civilisation.

3 L'objectif de ce travail est de présenter la philosophie de Ferran Adrià ainsi que la manière dont il intègre l'art, la créativité, la recherche scientifique et la recherche personnelle pour créer de nouveaux concepts, qui vont modifier également le sens du repas dans la vie humaine. Nous commencerons par rappeler quelques jalons de sa carrière pour analyser ensuite les clés de son succès dans le monde de la gastronomie, ainsi que son influence auprès du grand public.

\section{Le chemin vers la célébrité}

4 Nous commencerons ce parcours à partir de son entrée en contact avec El Bulli, restaurant situé sur le golfe de Roses (Girona-Espagne). ${ }^{1}$ Au début des années 60 , ce n'était qu'une buvette près de la plage, devenue par la suite un resto-grill, propriété d'un couple allemand. À partir de 1970, les propriétaires ont voulu introduire la cuisine française, et en 1976, il obtient sa première étoile Michelin, grâce au Français Jean-Louis Neichel. 
5 En 1983, le jeune Adrià qui accomplissait alors son service militaire, est venu faire un stage en cuisine pendant les vacances d'été. Le service militaire terminé, il a réintégré $\mathrm{El}$ Bulli en tant que second chef, aux côtés de Christian Lutaud. À cette époque, le restaurant fermait pendant les mois d'hiver, et les deux chefs ont profité de ce congé pour approfondir leur connaissance de la nouvelle cuisine française dans les restaurants les plus réputés.

6 En 1987, Ferran Adrià devient le chef d'El Bulli. C'est donc à partir de ce moment qu'il développera un style personnel et créatif dans la cuisine. En 1990, Ferran Adrià et Juli Soler, maître d'hôtel depuis 1981, deviennent propriétaires du restaurant, ce qui marquera un avant et un après dans l'histoire du restaurant. Outre la réfection de la cuisine, d'importants travaux de rénovation et de modernisation sont entrepris dans le restaurant : salles, parking, terrasses, jardins, etc. De plus, à partir de cette année-là, El Bulli restera fermé pendant six mois de l'année, d'octobre à mars, pour consacrer ce temps à la recherche et à la création. Dix ans plus tard, le souci de l'innovation et de la créativité amènera à n'ouvrir le restaurant que le soir. Le reste du temps, la cuisine devient un atelier de création et un laboratoire de recherche. Cet investissement portera ses fruits car à partir de 1990, les prix et récompenses se succèdent. ${ }^{2}$

7 El Bulli sera sélectionné comme «Meilleur restaurant du monde» selon The Restaurant Magazine à cinq reprises et pendant quatre années consécutives : 2002, 2006, 2007, 2008 et 2009. En 2010, le célèbre restaurant restera en deuxième position. En somme, en quinze ans, Adrià est arrivé au sommet de la nouvelle cuisine espagnole, constituant également un référent mondial dans le monde de la gastronomie. ${ }^{3}$

En 2004, La Fondation Alicia (Alimentation et Science), centre de recherche sur l'innovation technologique en alimentation, invite Ferran Adrià à collaborer comme Président du Conseil assesseur. ${ }^{4}$

En 2005, l'Université Camilo José Cela (Madrid) a créé la « Chaire Ferran Adrià » consacrée à la culture gastronomique et aux sciences de l'alimentation.

10 La consécration de son travail de créateur et de chercheur a été confirmée par le doctorat Honoris Causa décerné par plusieurs universités. En 2007, la Faculté de Chimie de l'Université de Barcelone, sous la proposition de Claudi Mans, est la première à accorder à un chef cette distinction. En 2008, c'est l'Université d'Aberdeen en Écosse, sous la proposition de Christopher Fynsk, du Centre de la pensée moderne, qui reconnaîtra le travail de l'Espagnol. Dans son discours de présentation, Fynsk compare Adrià à Picasso et à Miro, en soulignant le génie créateur du premier qui s'est révélé un penseur destiné à renouveler le lien entre l'alimentation intellectuelle et l'alimentation sensorielle de telle sorte qu'il est arrivé à changer pour toujours notre compréhension sur la relation entre cuisiner et manger. ${ }^{5}$ En 2010, le Département de Technologie des Aliments de l'Université Polytechnique de Valencia, lui octroie également cette distinction.

11 Non seulement les institutions universitaires reconnaissent son travail, mais plusieurs prix et bourses portent aujourd'hui son nom: Bourse International Ferran Adrià, de la Mairie de Girona et de la Generalitat de Catalogne ou le Prix Ferran Adrià de l'Université de Barcelone.

12 Pour conclure, Ferran Adrià est devenu une figure incontournable de la cuisine et de la gastronomie espagnoles non seulement au niveau national mais également mondial. 


\section{Ferran Adrià et les médias}

13 Sans doute, tous ces prix et ces reconnaissances dans le domaine spécifique de la gastronomie seraient passés inaperçus du grand public sans le concours des médias. La présence constante de Ferran Adrià dans des journaux et magazines a contribué à faire de lui une figure de premier ordre de la nouvelle cuisine espagnole.

C'est ainsi que le premier magazine à attirer l'attention sur lui en 1999 est «El Pais semanal », (supplément dominical de «El Pais»). Dans l'édition du 20 juin, Adrià apparaissait en couverture, qualifié comme le meilleur cuisinier du monde. ${ }^{6}$

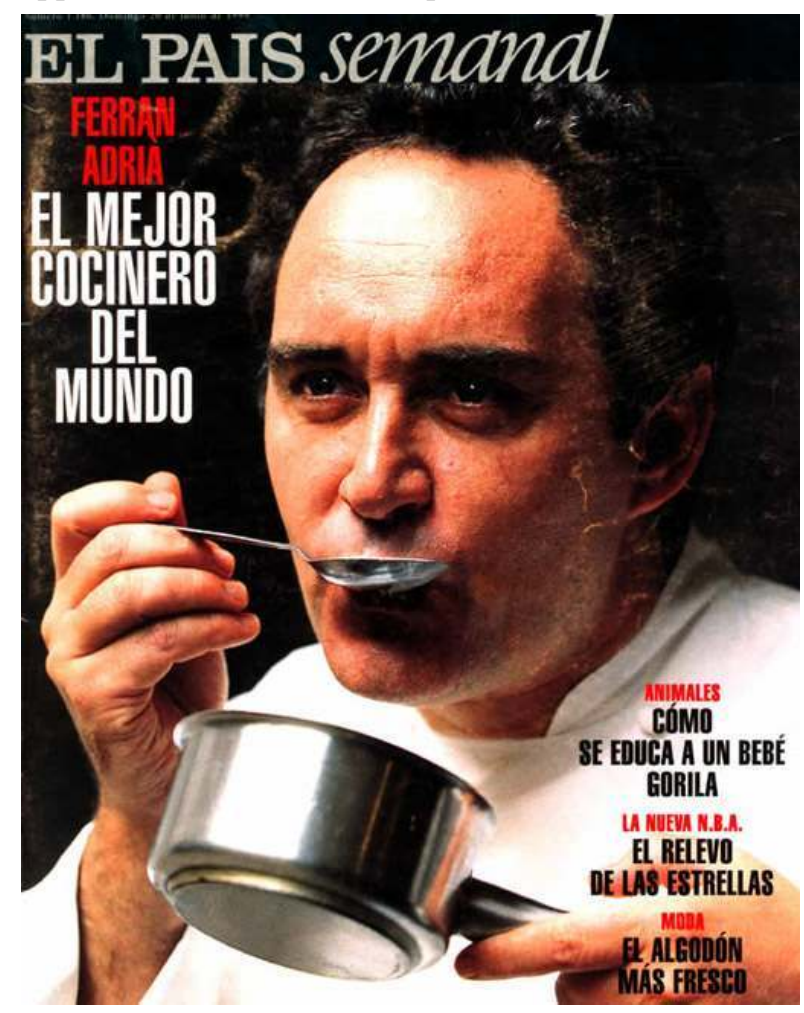

Couverture El Pais semanal, 20-06-1999

Dans ce reportage, le journaliste Agusti Fancelli présente les clés de la cuisine du chef espagnol à partir de trois interviews différentes. Adrià raconte avec beaucoup de simplicité son parcours professionnel jusqu'à son arrivée à El Bulli, et surtout le moment où il a compris qu'il devait développer un style personnel, créatif dans la cuisine. Fancelli met en exergue certaines de ses réalisations, telles que la « déconstruction de l'omelette de pommes de terre ", les espumas et les airs, le mélange de températures et de textures, dont nous parlerons un peu plus loin. Nous pouvons considérer cet article comme le début d'un véritable engouement envers le chef espagnol. Ce dernier fera l'objet de plusieurs reportages dans la presse internationale.

2000, la revue Time publiait un bref article qui cependant mettait bien en évidence l'originalité de El Bulli et de son chef dans les différentes combinaisons de textures, températures et saveurs. L'auteur mentionne quelques célèbres réalisations comme les « raviolis" liquides, les œufs de caille pochés et caramélisés ou encore la crème glacée aux amandes accompagnée d'un soupçon d'huile à l'ail et de vinaigre balsamique. El Bulli est qualifié de «lieu de pèlerinage» pour les amateurs de repas non 
conventionnels, faisant ainsi référence à son menu de dégustation composé de nombreux petits plats. L'article se termine en soulignant la « recette » d'Adrià, laquelle consiste à être " froid et méthodique ", à voyager, à tester et à énormément lire, afin de se renouveler sans cesse et de rompre avec la monotonie des sentiers battus. ${ }^{7}$

En 2003, The New York Times Magazine (le 10 août 2003) consacrait à la nouvelle «nouvelle cuisine » espagnole, un long reportage, intitulé A Laboratory of Taste. ${ }^{8}$

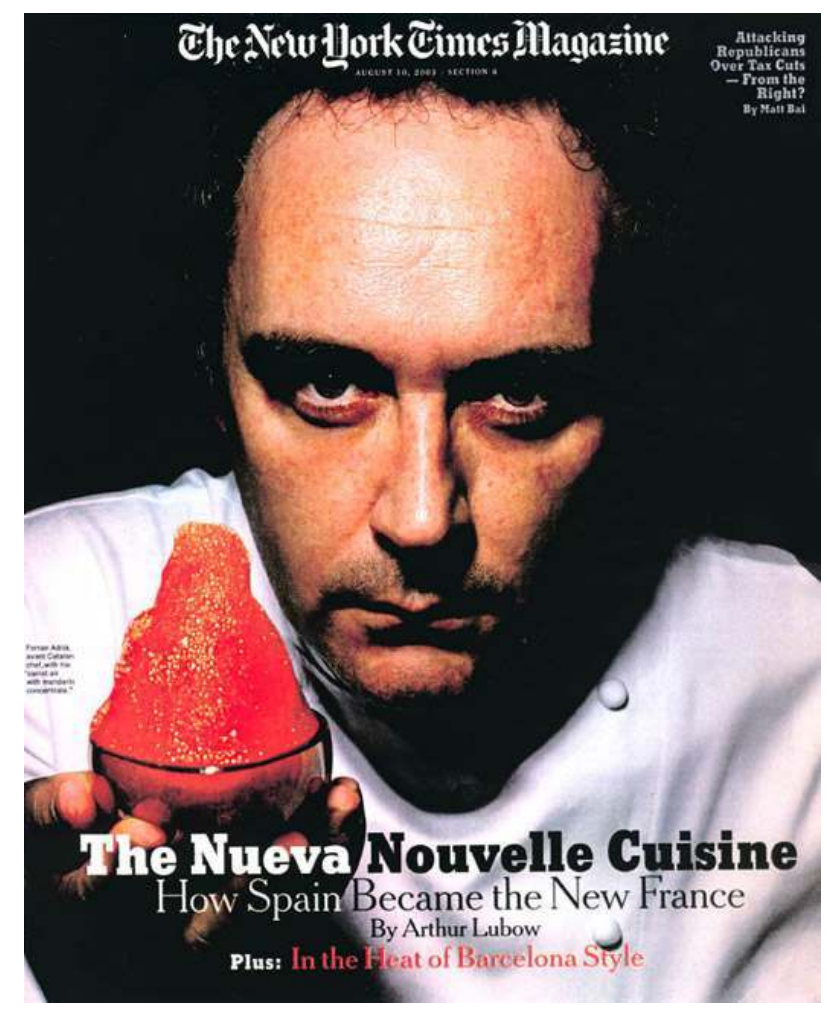

The New York Times Magazine, 10-8-2003

L'article d'Arthur Lubow dévoilait que, selon Marc Veyrat, chef étoilé de la cuisine française, la gastronomie d'avant-garde n'était plus française mais espagnole. Le journaliste, piqué sans doute par la curiosité, en avait fait l'expérience lui-même. Après un voyage en Europe, il constatait que depuis vingt ans, rien n'avait changé dans la cuisine française. Ce qui en 1970 était révolutionnaire, ne l'était plus. En Espagne en revanche, tant en Catalogne qu'au Pays Basque, une nouvelle conception de la gastronomie voyait le jour. Et d'ajouter le nom de celui qui était sur toutes les lèvres : Ferran Adrià.

El Bulli est présenté par Lubow comme la « Mecque des gourmets » et Adrià, comme le plus imaginatif des chefs de toute l'histoire. De son passage à El Bulli et de son menu de dégustation surprenant, le journaliste se souvient en particulier d'un plat qu'il considère être le plus sublime: un tableau constitué de sept blocs de gélatine chaude ayant l'apparence d'une aquarelle car tout en nuances. En somme, une «nouvelle cuisine » par la nouvelle technique et les nouveaux concepts. Il souligne ses trouvailles innovantes et osées, en faisant remarquer l'alliance entre cuisine et création artistique. Des commentaires de Lubow, il ressort que l'on entre dans El Bulli comme dans un musée, une galerie d'art, ou dans une salle de cinéma d'art et essai. Aussi l'on n'y va pas pour s'y restaurer mais pour faire une expérience esthétique. ${ }^{9}$ 

quand déjà dans les années 1990, il déclarait concevoir le menu de dégustation comme un film qui relate une histoire, ce qui explique qu'il se renouvelait chaque année car il est impossible de raconter tous les ans une même histoire, ni projeter le même film..$^{10} \mathrm{Le}$ reportage eut un grand écho et déchaîna de nombreuses réactions dans la presse internationale, notamment en France. ${ }^{11}$ caille caramélisés -, reconnaissant le caractère révolutionnaire de son art culinaire ainsi que sa première place dans la gastronomie mondiale. El Bulli est qualifié de "Saint Jacques de Compostelle des pèlerins gourmands ». Le reportage transmet également les commentaires de certains chefs français tels que Joël Robuchon et Pierre Gagnaire, sans doute le plus critique à l'article de The New York Times.

Enfin, en 2004, Time inscrivait Ferran Adrià sur la liste des 100 personnes les plus influentes de l'année sous la rubrique « Artists \& Entertainers ». Ce magazine considérait Ferran Adrià comme le chef le plus innovant du moment. Sa cuisine y est décrite comme une « alchimie » où se retrouvent unis «le sucré et le salé, le froid et le chaud, le familier et les cuisines du monde ». Dans un souci de dépassement de lui-même, mais aussi dans le but de surprendre, Adrià propose à El Bulli un menu de dégustation constamment renouvelé. L'article se termine par la comparaison entre la découverte de l'Amérique par l'Espagne et l'esprit de Ferran Adrià pour conquérir de nouveaux espaces culinaires. ${ }^{13}$

Depuis ce jour, les articles des journaux espagnols et étrangers, qui présentent la figure et la cuisine de Ferran Adrià, tant en version papier que on line, sont innombrables. ${ }^{14}$

\section{La révolution culinaire d'Adrià}

Comment expliquer cette popularité rarement atteinte par un chef ? Dans quelle mesure, peut-on considérer les innovations culinaires d'Adrià comme une révolution dans le monde de la gastronomie?

La réponse à ces questions se trouve probablement dans la démarche adoptée par notre cuisinier. Si pendant les premières années, Adrià continue de pratiquer la cuisine française, c'est lors d'un congrès à Nice, en 1986, qu'il découvre le point de départ d'une cuisine personnelle et créative. Dans une des sessions, le chef Jacques Maximin répond à une question par une phrase simple: "la créativité est ne pas copier ${ }^{15}$. À partir de ce moment, Adrià cherchera sans cesse à se renouveler et à se perfectionner, en s'inspirant du monde qui l'entoure. Ainsi, il établira des alliances heureuses entre Cuisine et Art, Cuisine et Science, Cuisine et Technique, ce qui lui permettra d'atteindre son but: Cuisine et Bonheur.

\section{Cuisine et Art : « La créativité est ne pas copier »}

À partir de ce principe très simple mais difficile à tenir dans le monde actuel, Adrià a développé un style personnel dans le monde de la gastronomie. Le défi était de s'affranchir de la cuisine espagnole traditionnelle et de la nouvelle cuisine française, sans 
les abandonner mais en incluant des éléments des cuisines du monde, et en particulier les cuisines chinoise et japonaise.

En 1992, pendant les mois de fermeture annuelle de El Bulli (six mois), Adrià a commencé à travailler dans l'atelier de son ami sculpteur Xavier Medina Campeny. En voyant travailler l'artiste, il comprit que la créativité en cuisine consistait à préparer de nouveaux plats à partir des sensations qui mettaient à contribution tous les sens : la vue pour les couleurs et les formes, l'odorat, le toucher dans les nouvelles textures et les mélanges de température... dans le but de créer l'émotion et pas seulement le plaisir gustatif.

Ce contact direct avec le monde de l'Art lui a permis d'introduire de nouvelles tendances d'avant-garde comme celle de la déconstruction. Appliquée à la gastronomie, la déconstruction présente les ingrédients d'un plat de façon inhabituelle, créant la surprise du convive qui ne reconnait pas à sa vue le plat traditionnel mais en retrouve toute la saveur, l'arôme et la couleur. Dans la déconstruction, les ingrédients sont conservés, mais en transformant leur texture et parfois également la température. L'exemple le plus célèbre est celui de la déconstruction de l'omelette de pommes de terre, plat traditionnel espagnol et apprécié par $90 \%$ de la population.

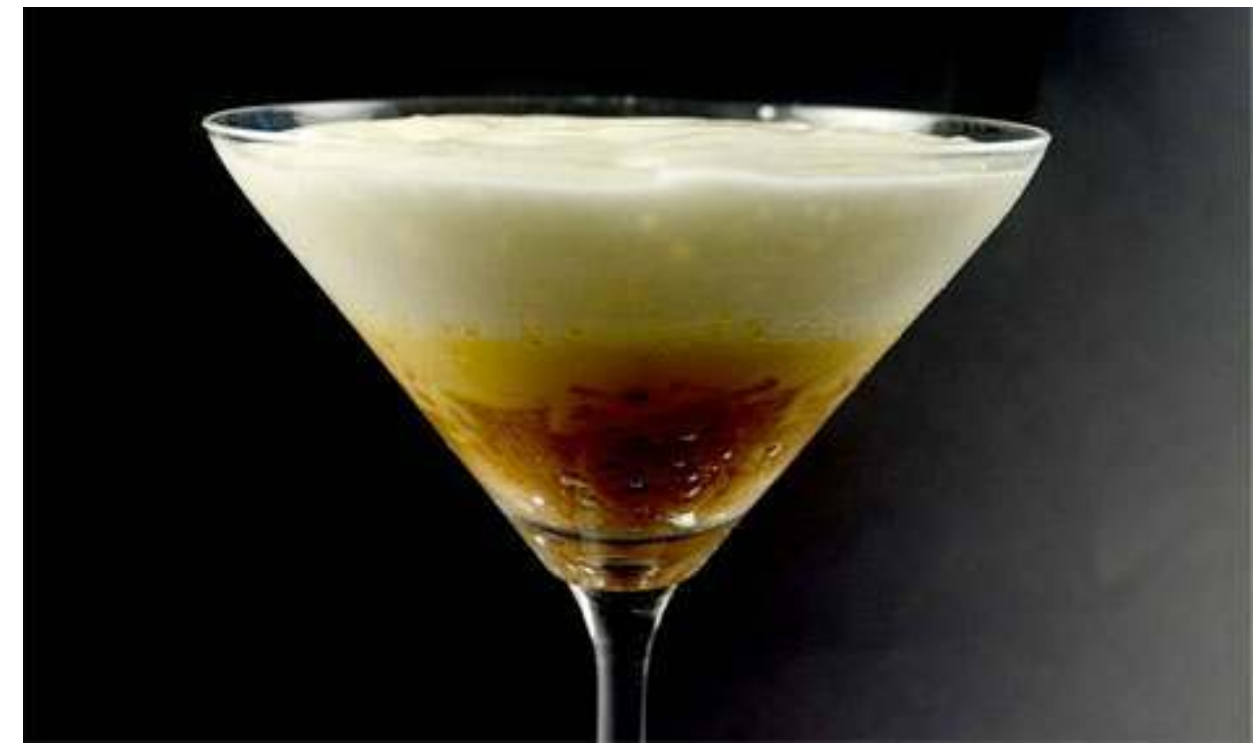

Déconstruction de l'omelette de pommes de terre

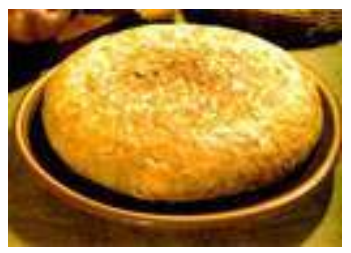

Omelette traditionnelle

Un autre exemple emblématique est la déconstruction de l'orange. Nombre de ses plats dénommés "en textures » sont des déconstructions de plats traditionnels tels que le panaché de légumes ou le poulet au curry.

C'est ainsi que la cuisine est devenu l'atelier de l'artiste : El Bulli-Taller. À partir de 1994, Adrià a formé une équipe créative qui travaillait durant la moitié de l'année à élaborer de nouvelles réalisations culinaires ${ }^{16}$. 
Une confirmation de l'alliance entre Cuisine et Art se trouve dans l'invitation à participer à l'exposition Feria Documenta 12 de Kassel en $2007 .{ }^{17}$ El Bulli était le pavillon G de cette exposition.

En dépit des critiques émises sur les méthodes employées par Adrià, nul ne met en doute que son restaurant représentait le fer de lance de la cuisine d'avant-garde, créant ainsi le nouveau langage de la gastronomie.

\section{Cuisine et Science : nouveaux concepts et nouvelles techniques}

mière démarche pour renouveler le monde de la gastronomie a été artistique, Adrià a rapidement compris que la transformation des aliments passait par la connaissance de leurs propriétés physiques et chimiques. Une fois de plus, le contact avec des scientifiques lui a ouvert de nouveaux horizons. En 2003, l'équipe créative s'est donc enrichie de l'apport de plusieurs scientifiques et l'atelier de l'artiste est devenu également un laboratoire d'expérimentations grâce au département scientifique de El Bulli Taller. ${ }^{18}$ Dans un esprit de véritable chercheur, Adrià a relevé le défi en dépassant les connaissances acquises pour élaborer de nouveaux concepts et appliquer de nouvelles techniques qui étaient irréalisables jusqu'alors. Comme le chef le rappelle, un nouveau concept n'est pas une nouvelle recette. La nouvelle recette consiste en un nouveau mélange d'ingrédients et de saveurs. Le nouveau concept, en revanche, passe par la transformation des ingrédients dans de nouvelles textures, formes et températures.

Cette recherche scientifique a introduit des composantes naturelles - gélifiants, émulsifiants et autres - pour altérer les propriétés physiques des ingrédients. ${ }^{19}$ Elle a été accompagnée également d'une recherche technique et technologique de design industriel pour créer de nouveaux outils (sniffs, pipettes, cuillères), ou récupérer pour la cuisine des instruments utilisés ailleurs tels que le siphon Isi. ${ }^{20}$

6 Grâce à ces recherches, l'offre gastronomique de El Bulli s'est diversifiée en espumas (écumes), airs, nuages, sphérifications, nouvelles textures, gélatines chaudes, dont nous pouvons citer l'air de carotte à la mandarine, les sucettes aux légumes, les croquettes et les ravioli liquides, le caviar de thé vert, de pomme, de melon, les olives liquides, etc. ${ }^{21}$

\section{Le menu de dégustation}

37 Cette rupture avec la cuisine et le repas traditionnel donnera lieu à une nouvelle façon de présenter les menus là où les frontières entre le salé et le sucré, le chaud et le froid s'estompent. À partir de l'année 2006, Adrià proposera à ses clients uniquement un menu de dégustation composé de 35 plats, qui ne font qu'une bouchée, en s'inspirant de la cuisine minimaliste japonaise, mais aussi des traditionnelles tapas espagnoles. Chaque plat est servi avec ses propres instructions afin de le déguster de la meilleure façon. Ce menu sera renouvelé chaque année jusqu'à la fermeture du restaurant en 2011. Les plats sont structurés de la façon suivante :

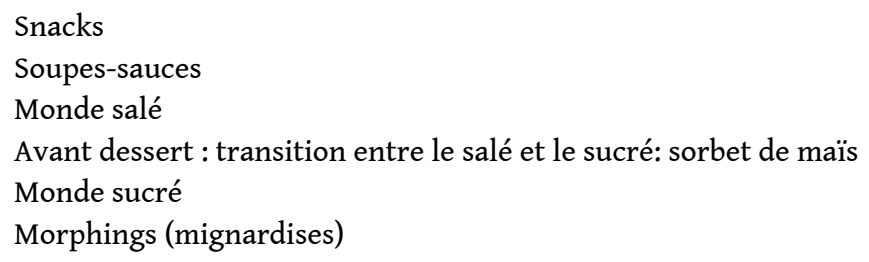


En 2007, le menu de dégustation a inspiré une œuvre musicale Le livre des illusions du compositeur français Bruno Mantavoni, qui fut créée en juin 2009 et jouée à la Salle Pleyel à Paris. ${ }^{22}$ Cette œuvre se compose de 35 mouvements, un pour chaque plat, à travers lesquels le musicien illustre la texture de chacune des recettes du menu. À titre d'exemple, voici la liste de mouvements-plats :

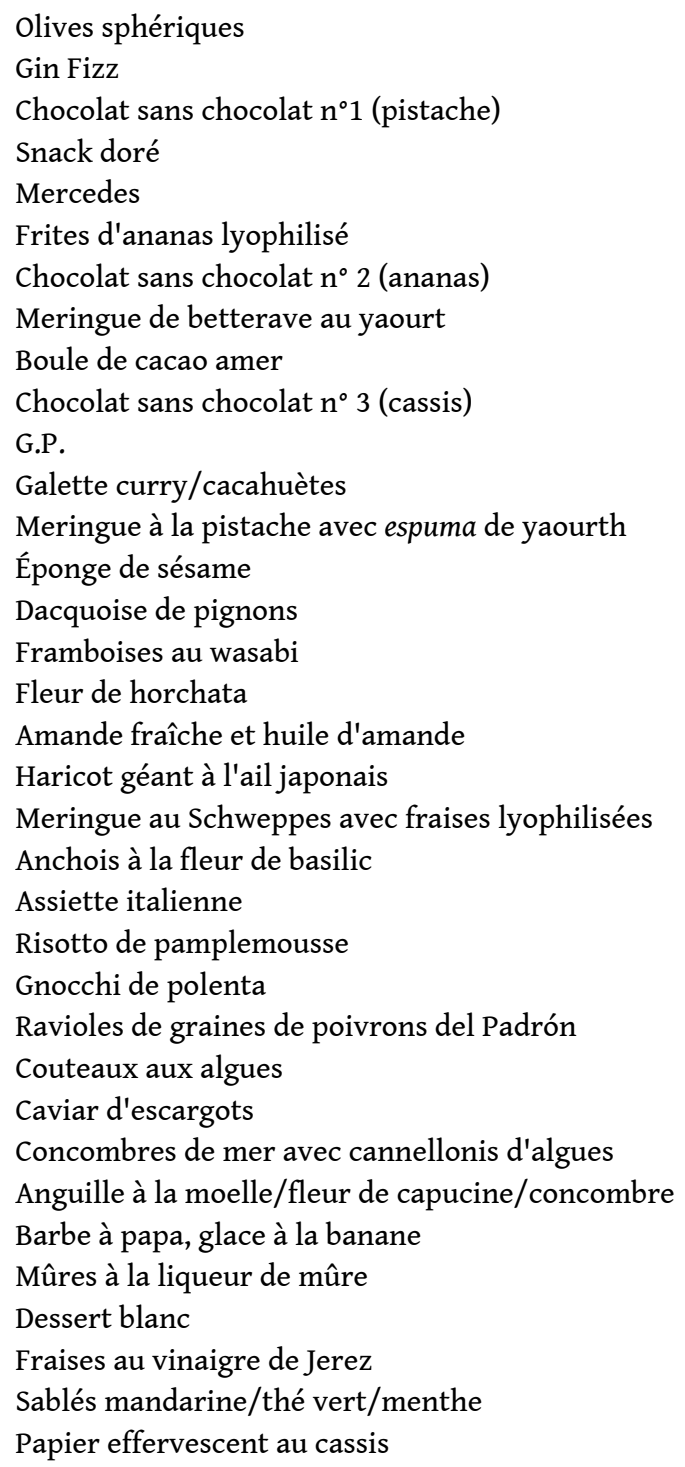

Pour conclure, dans la cuisine d'Adrià, aucun ingrédient n'est privilégié : homard, foiegras, truffes, pour faire une cuisine raffinée, qui procure à la fois un plaisir à la plupart de nos sens : la vue, l'odorat, le goût et le toucher. Tous les produits naturels, de la terre comme de la mer, ont la même valeur, indépendamment de leur prix et de leur prestige.

\section{La philosophie de Ferran Adrià}

Adrià et son équipe sont conscients d'avoir créé une nouvelle philosophie de la cuisine et de l'alimentation présentée pour la première fois de manière synthétique en janvier 2006, lors du concours « Madrid Fusion ». ${ }^{23}$ Il est intéressant de s'attarder sur le premier point : 
41 La cuisine est un langage à travers lequel on peut exprimer l'harmonie, la créativité, le bonheur, la beauté, la poésie, la complexité, la magie, l'humour, la provocation, la culture. 24

Cette façon de concevoir la cuisine : l'élaboration des plats ainsi que leur présentation et par conséquent l'alimentation, élève le simple besoin biologique qu'est celui de manger, à une réalisation humaine qui pourrait être aussi bien du domaine de l'art que de la science.

C'est pourquoi lorsqu'on s'attarde un peu aux différents éléments qui composent le langage de Ferran Adrià, nombre d'entre eux font référence à l'Art, d'autres aux émotions, et l'ensemble à toute la personne humaine, pas seulement à l'estomac ou aux papilles gustatives.

44 «L'harmonie, la créativité, la beauté, la poésie » renvoient au monde de l'Art dans lequel la création artistique rejoint le plaisir esthétique du spectateur, créant ainsi un lien communicatif entre l'artiste et son public. «La provocation », telle qu'elle est dans l'Art d'avant-garde tend à briser les tabous, à rompre les clichés, à ouvrir de nouveaux chemins d'expression.

De plus, la "complexité » demande une recherche tenace afin de trouver les solutions appropriées aux défis. Tout comme le scientifique ou l'alchimiste du Moyen-Âge, qui cherchait la pierre philosophale, derrière les réalisations de Ferran Adrià, se cachent autant de nombreuses expériences que d'heures de travail acharné.

Dans la cinquième partie du catalogue, un chapitre intitulé « Raison » fait référence au cerveau et établit la liste d'émotions et de réactions que la cuisine d'Adrià tend à susciter. Par exemple: "Transgression, ironie, provocation, souvenirs d'enfance, surprise, jeu, magie, décontextualisation, tromperie, mémoire et culture gastronomiques, harmonie $» .^{25}$ Cette liste vient compléter les éléments cités précédemment.

Comme nous pouvons le constater, Adrià accorde une grande importance à l'aspect ludique de la cuisine et des repas, aux souvenirs d'enfance, et au propre background culturel et gastronomique. Dans «l'humour et l'ironie», nous pouvons trouver les émotions humaines qui peuvent se tisser, se développer autour d'un repas, et qui nous font oublier la grisaille de la vie quotidienne, tandis que dans « la provocation et la transgression » nous éprouvons des émotions intellectuelles, provoquées par la rupture d'avec les menus et les plats traditionnels.

Comme exemple de plat ludique, nous pouvons mentionner le plat des épices: une soupesauce sur laquelle les différentes épices sont placées comme les chiffres d'une montre. Il faut les manger l'une après l'autre dans le sens des aiguilles, et savoir les reconnaître.

Finalement, le mot qui définit le mieux la cuisine de Ferran Adrià est « la magie ${ }^{26}$. En effet, la magie est la surprise créée par l'inattendu, l'impossible, ce qui défie les lois de la nature et nous fait rêver, ce que l'on n'a jamais vu... Nombre de ceux qui ont eu la possibilité de dîner à El Bulli décrivent leur expérience et leur émotion les comparant à de la magie.

Tous ces ingrédients pourraient se concentrer dans l'une des maximes d'Adrià : le but de la cuisine est le bonheur. Le bonheur du cuisinier à l'heure de concevoir et de réaliser les plats, et le bonheur de ceux qui ont la chance de les déguster. Certes, un bonheur éphémère que l'on pourrait résumer comme les bons moments de la vie, mais qui laissent la trace d'un souvenir bien plus grand que celui d'un simple bien-être produit par un repas délicieux. 


\section{Impact sur la société espagnole}

51 Mais tout cela, ne serait qu'une cuisine de luxe pour une élite privilégiée sans la personnalité de Ferran Adrià et de son équipe. Outre sa présence dans les médias, les prix et les distinctions, ce qui a fait d'Adrià un chef populaire et aimé des espagnols est le fait d'avoir joué la carte de la transparence. Beaucoup de chefs gardent le secret de leurs trouvailles pour continuer à être les seuls à élaborer telle ou telle recette. Adrià, en revanche, non seulement a formé les meilleurs chefs du monde, qui sont passés par El Bulli, mais il a encore voulu diffuser ses recettes et ses découvertes afin que le plus grand nombre en profite. À côté de certaines techniques, qui restent sophistiquées et demandent des outils particuliers, d'autres au contraire peuvent être réalisées à la maison et s'introduire dans la cuisine familiale. Ainsi il est chef d'une cuisine d'avantgarde à El Bulli, mais aussi de la cuisine facile à la maison. Comme il le déclare lui-même dans une interview à Muñoz Lara de El País :

Ce n'est pas vrai que la haute cuisine n'a pas percé dans la société. Le menu de dégustation de El Bulli requiert une technologie qui n'existe pas à la maison, mais nous avons publié un livre pour l'adapter à la logistique du foyer. Cela veut dire que la technologie est uniquement un outil; ce qui est important est avoir de l'imagination. ${ }^{27}$

52 Dans ce sens, le titre de l'un de ses premiers livres, paru en 1998, est révélateur «Les secrets de El Bulli ». De plus, il a édité sous un format audiovisuel les catalogues de toutes les réalisations du fameux restaurant pendant dix ans (1999-2009). ${ }^{28}$ Chacun des livres ne se réduit pas à un simple recueil de recettes, mais il exprime la façon de concevoir la cuisine.

53 Ses livres de cuisine et la série de 11 DVD, La cocina fácil de Ferran Adrià, montrent également sa volonté de mettre son expérience au service de la cuisine familiale. ${ }^{29} \mathrm{En}$ ce sens, Adrià parle d'une «nouvelle cuisine familiale » qui introduirait à la maison non les techniques de El Bulli, mais sa philosophie. Les recettes proposées par Adrià dans cette série documentaire parue en 2004, sont simples et rapides, car il est conscient qu'on n'a pas beaucoup de temps à la maison pour cuisiner. En même temps, il s'agit d'une cuisine innovante et imaginative, avec une présentation agréable et soignée. De plus, Adrià donne des conseils et partage les principes qui ont inspiré sa révolution culinaire.

Son dernier ouvrage, La comida de la familia (Barcelona, RBA Libros 2011), propose les recettes de repas cuisinés pour « la famille de El Bulli », c'est-à-dire l'équipe qui travaillait dans le célèbre restaurant et qui mangeait un repas très différent de ce qu'ils préparaient pendant toute la journée pour les clients qui arrivaient le soir. C'était un repas bon, simple et bien préparé. Il s'agit donc d'un livre de cuisine facile ; 31 menus pratiques pour cuisiner à la maison, avec des produits simples que l'on trouve dans le supermarché et accessibles à un petit budget (autour de 4 euros). ${ }^{30}$ Dans ce dernier ouvrage, Adriá propose une cuisine créative à la portée de tous, même en temps de crise. Les menus et les recettes peuvent s'adapter également à des collectivités de plus de 20 convives. En plus des recettes, le chef livre des conseils sur la manière d'organiser la réserve alimentaire, planifier ses courses et sur la gestion d'une cuisine familiale ou professionnelle dont l'un des points essentiels est celui de l'organisation. ${ }^{31}$

Adria est également relié à la cuisine espagnole traditionnelle et familiale à travers les tapas. Ces en-cas traditionnels ont connu une évolution dans la gastronomie espagnole, du 
simple apéritif au repas informel, en famille ou le plus souvent entre amis. C'est non seulement une façon de manger, mais aussi un style de vie marqué par la convivialité. Ce qui caractérise la tapa est le fait de pouvoir la manger souvent avec les doigts, en une ou deux bouchées et de ce fait, on peut en déguster plusieurs au cours d'un repas. Elles se sont même exportées à l'étranger, non seulement dans les bistrots et les tavernes touristiques mais aussi dans les restaurants. On pourrait même se demander si nous n'assistons pas à une nouvelle phase de cette évolution, du repas informel au repas tout court.

Une manifestation de l'importance croissante des tapas dans la gastronomie mondiale est l'exposition itinérante « Tapas. Spanish Design for Food " ${ }^{32}$.

Après l'annonce de la fermeture de El Bulli en tant que restaurant, Ferran Adrià s'est associé à son frère cadet Albert pour s'investir dans le monde des tapas et des cocktails. ${ }^{33}$ Albert Adrià, qui s'était incorporé à l'équipe en 1985 dans la section de pâtisserie, avait ouvert en 2008 son propre établissement à Barcelone, Inopia Classic Bar, consacré aux tapas traditionnelles mais aussi d'avant-garde, en essayant de concilier tradition et modernité. ${ }^{34}$ Cette nouvelle tendance de la cuisine d'avant-garde appliquée aux tapas a donné lieu à ce qu'on appelle les "gastrobars ». En 2011, les frères Adrià en association avec les frères Iglesias ont ouvert, également à Barcelone, $41^{\circ}$, un bar dédié initialement aux cocktails et aux snacks, mais qui très vite est devenu un petit restaurant de 16 places, le «MiniBulli », avec un menu de snacks accompagnés de cocktails. ${ }^{35}$ Quelques mois plus tard, ils ont ouvert Tickets, un nouvel espace à côté de $41^{\circ}$ consacré au monde des tapas, baptisé comme le "Bulli du quartier $»{ }^{36}$ Dans cet espace, nous retrouvons l'aspect ludique des réalisations de El Bulli : la déco, les uniformes des serveurs et la carte des tapas évoquent le spectacle du Cirque.

\section{Conclusion}

58 Ferran Adrià a introduit un avant et un après dans le monde de la gastronomie. Non seulement par ses réalisations d'avant-garde, alliant l'art et la science à l'élaboration culinaire, mais également et surtout par sa façon de concevoir le travail du cuisinier, non comme une tâche purement manuelle, mais comme une activité humaine où toutes les facultés sont mises à contribution.

Il a été reconnu par ses pairs comme le chef le plus imaginatif et le plus créatif de la cuisine moderne. Entre 1987 et 2011, il a créé 1.846 plats. En ce sens, il est un exemple constant de dépassement de soi.

De plus, Adrià a réussi à introduire sa cuisine non seulement dans les médias, mais aussi à l'Université, dans les musées et les galléries d'Art, dans les hôpitaux, dans les foyers et dans les bistrots. Et cela non seulement en raison de la perfection ou de la qualité de ses recettes, mais aussi parce qu'il a su montrer l'importance du temps que nous consacrons à manger et du repas, qui peut être bon, attrayant, amusant, détendant, sans être forcément très cher. D'une grande souplesse, il a su s'investir dans la haute-cuisine d'avant-garde, comme dans la cuisine familiale et traditionnelle des tapas pour les renouveler. L'engouement en Espagne pour la cuisine créative et d'avant-garde qui s'introduit dans les foyers et dans les bars, doit sans doute beaucoup à son rayonnement.

61 À l'heure actuelle, Adrià n'est plus derrière les fourneaux, mais il est devenu l'ambassadeur de sa philosophie, qui a révolutionné le monde de la gastronomie, et de son 
nouveau projet : la Fondation El Bulli. Ce que Ferran Adrià veut transmettre aujourd'hui n'est pas seulement la partie technique de l'élaboration des aliments, mais les valeurs humaines qui sont transmises par la cuisine et pendant les repas. ${ }^{37}$

\begin{tabular}{|c|c|c|}
\hline 1990 & Deux étoiles & Michelin \\
\hline 1992 & $\begin{array}{l}\text { Prix National de Gastronomie } \\
\text { au meilleur Chef de Cuisine }\end{array}$ & Académie Nationale de gastronomie \\
\hline 1994 & Grand Prix de L'Art de la Cuisine & Académie Internationale de Gastronomie \\
\hline 1995 & El Bulli19/20 & Guide Gault \& Millau \\
\hline 1995 & El Bulli 9,75/10 & Guide espagnol Lo mejor de la gastronomía: \\
\hline 1996 & Clé d'Or de la Gastronomie. & Gault-Millau \\
\hline 1996 & Restaurant de l'année, & Club de Gourmets \\
\hline 1997 & Trois étoiles & Michelin \\
\hline 1997 & $\begin{array}{l}\text { Médaille d'Argent au mérite } \\
\text { Touristique de Catalogne. }\end{array}$ & Generalitat de Catalogne \\
\hline 1999 & Prix Giorgio Fini, & Città di Modena \\
\hline 1999 & Meilleur Chef de l'année, & Gourmetour \\
\hline 2002 & Creu de Sant Jordi, & Generalitat de Catalogne \\
\hline 2002 & $\begin{array}{l}\text { Médaille d'or au Mérite } \\
\text { Touristique. }\end{array}$ & Gouvernement d'Espagne \\
\hline 2002 & $\begin{array}{l}\text { Médaille d'or au Mérite } \\
\text { Touristique, }\end{array}$ & Generalitat de Catalogne \\
\hline 2003 & Silver Spoon. & Food Arts \\
\hline 2003 & $\begin{array}{ll}\text { Prix } & \text { protagonistes: } \\
\text { Gastronomie. } & \end{array}$ & Onda Cero Radio. Emisión de Luis del Olmo. \\
\hline 2003 & Catalan de l'année. & "El Periódico de Catalunya" \\
\hline 2003 & Prix Ciutat de Barcelona, & Ville de Barcelone. \\
\hline 2004 & $\begin{array}{l}\text { Ambassadeur de la Marque } \\
\text { Espagne, catégorie culture. }\end{array}$ & $\begin{array}{l}\text { Forum de Marques réputées espagnoles (Ministère de } \\
\text { l'Industrie, du Commerce et du Tourisme. Institut } \\
\text { Espagnol du Commerce Extérieur). }\end{array}$ \\
\hline
\end{tabular}




\begin{tabular}{|c|c|c|}
\hline 2004 & $\begin{array}{l}\text { Fils préféré de la Ciudad de } \\
\text { Hospitalet. }\end{array}$ & Ville de Hospitalet de Llobregat. \\
\hline 2005 & $\begin{array}{l}\text { Preisträger Ferran Adrià } \\
\text { Sektion Große Kochkunst. }\end{array}$ & Witzigmann Preis \\
\hline 2007 & $\begin{array}{l}\text { Médaille d'Or au mérite de } \\
\text { Beaux Arts. }\end{array}$ & Ministère de la Culture d'Espagne \\
\hline 2007 & Award & The White Guide Gastronomy \\
\hline 2007 & $\begin{array}{l}\text { Médaille d'Or au Mérite } \\
\text { Européen. }\end{array}$ & Fondation du Mérite Européen, UE \\
\hline 2008 & Prix National de l'Hôtellerie. & Fédération espagnole d'Hôtellerie et restauration. \\
\hline 2008 & Prix don Quichotte. & Académie de Gastronomie de Castilla-La Mancha \\
\hline 2009 & Grande Croix du Mérite Civil. & Conseil de Ministres d'Espagne \\
\hline 2009 & Meilleur chef. Prix Augie & Culinary Institute of America \\
\hline 2010 & Meilleur chef de la décennie & The Restaurant Magazine \\
\hline 2010 & $\begin{array}{l}\text { Presidente del Basque } \\
\text { Culinary Center }\end{array}$ & \\
\hline
\end{tabular}

\section{NOTES}

1. Les informations sur Ferran Adrià et El Bulli proviennent du site web du restaurant : http:// www.elbulli.com/historia/index.php?lang=es\&seccion=2. Consulté le 30 septembre 2013.

2. Voir annexe 1.

3. Ainsi, il n'est pas étonnant que le dîner de gala offert la veille des noces entre le prince des Asturies, Philippe de Bourbon, et doña Letizia lui soit confié à côté d'autres grands chefs espagnols.

4. Le centre a été créé en 2004 sous le patronage de la Generalitat de Catalogne et de la Fondation Catalunya-La Pedrera. Il est destiné à promouvoir les bonnes habitudes alimentaires et la mise en valeur du patrimoine agroalimentaire et gastronomique.

5. http://www.elbulli.com/historia/version_imprimible/1961-2011_es.pdf. Consulté le 30 septembre 2013.

6. El Pais semanal, 20 juin 1999.

7. http://content.time.com/time/magazine/article/0,9171,59799,00.html\#ixzz2gw74p1YO. Consulté le 5 octobre 2013. 

pagewanted=all\&src=pm. Consulté le 7 octobre 2013.

9. L'ouïe est le seul sens qui n'est pas sollicité par la cuisine d'Adrià car comme il le dit lui-même « les asperges ne chantent pas ».

10. El Pais semanal, 20-6-1999, p. 24.

11. Deux jours plus tard, le 10-8-2003, le quotidien El Mundo publiait un compte rendu de l'article de The New York Times. http://www.elmundo.es/elmundo/2003/08/10/ sociedad/1060501524.html. The Daily Telegraph, 5-9-2003. The Canadian Press, 19-01-2004, etc. Pierre Gagnaire réagissait aux critiques du journal nord-américain à la cuisine française.

12. Le Monde 2, 24-1-2004.

13.

http://content.time.com/time/specials/packages/ article/0,28804,1970858_1970890_1971358,00.html. Consulté le 5 octobre 2013.

14. Plus de 2.500 articles et plus de 14.000 pages sur Ferran Adrià. http://press.elbulli.com/? lang=es, consulté le 25 août 2014.

15. Agustí Fancelli, "El mejor cocinero del mundo", http://cultura.elpais.com/ cultura/2011/11/24/actualidad/1322089226_850215.html. Consulté le 9 octobre 2013.

16. http://www.elbulli.com/historia/index.php?lang=es\&seccion=3\&subseccion=8. Consulté le 30 septembre 2013.

17. Feria Documenta est une exposition qui a lieu chaque année à Kassel consacrée à l'art contemporain.

18. http://www.elbulli.com/historia/index.php?lang=es\&seccion=6\&subseccion=2. Consulté le 30 septembre 2013.

19. Parmi les additifs utilisés dans la cuisine de Ferran Adrià nous pouvons mentionner la lécithine de soja, le calcium, les alginates, des gélifiants à base d'algues, des produits épaississants, des fruits lyophilisés, du nitrogène liquide et autres produits selon le résultat recherché de différentes textures : sphérification, gélatines, émulsions, etc. Cette technique a été très critiquée par un autre chef espagnol, Santi Santamaria, créant la polémique dans les médias. Toutefois, la renommée et la popularité de Ferran Adrià l'ont emporté sur ses détracteurs.

20. Actuellement en vente chez tous les commerçants en électroménager.

21. L'espuma a la texture de la mousse du bain, non celle de la mousse au chocolat. Pour obtenir la mousse traditionnelle, il est nécessaire d'ajouter les blancs d'œuf battu en neige et ou la crème fraîche, tandis que pour l'espuma, on introduit le jus de fruit gélifié dans le siphon Isi auquel on ajoute de l'air. Le résultat est une nouvelle texture aérienne. La procédure pour obtenir de l'air, aire, est très simple. Dans un récipient contenant du jus de légume ou de fruit, on introduit un mixeur électrique et on obtient de l'air aux différents parfums.

22. http://www.youtube.com/watch?v=Ec4b-GtzRBM. Consulté le 1 décembre 2013.

23. Le concours Madrid Fusion est un rassemblement de 60 journalistes du monde entier qui établissent la liste de chefs les plus influents de l'année. Le premier nom de la liste était celui de Ferran Adrià suivi d'Alain Ducasse et de Juan Mari Arzak.

24. La cocina es un lenguaje mediante el cual se puede expresar armonía, creatividad, felicidad, belleza, poesía, complejidad, magia, humor, provocación, cultura. http://www.elbulli.com/ historia/index.php?lang=es\&seccion=7\#. Consulté le 30 septembre 2013. Traduit par nos soins.

25. Transgresión, ironía, provocación, recuerdos de la infancia, sorpresa, juegos, magia, descontextualización, engaño, memoria, cultura, gastronomía, armonía.

26. C'est le titre de l'ouvrage consacré à Ferran Adrià par Manfred Weber-Lamberdière, Ferran Adrià. El mago de elBulli, traduction espagnole, Madrid, Aguilar, 2010.

27. http://sociedad.elpais.com/sociedad/2011/12/08/actualidad/1323369047_641179.html

28. Série de 10 DVD sous le titre Histoire d'un rêve.

29. Le nombre de sites, vidéos sur YouTube et blogs qui montrent la déconstruction de l'omelette de pommes de terre est un échantillon de la popularité de la cuisine de Ferran Adrià. 
30. Le livre a été publié en anglais sous le titre The family meal. Home cooking with Ferran Adria, Phaidon Press, 2011.

31. Un signe de l'aura médiatique d'Adrià et de son impact dans les foyers espagnols est le fait que le livre a été lancé également sous forme d'application pour Ipad et Tablettes, créée par Telefonica sous le titre: Adria en casa (Adrià à la maison). La présentation de la nouvelle application a été diffusée par internet via terra.es le 30 novembre 2012.

32. L'exposition de plus de 200 pièces, organisée par Acción Cultural Española (AC/E) et Spain, Arts \& Culture, avec la collaboration de designers, architectes et cuisiniers a déjà été présentée à Tokyo (26 octobre-4 novembre 2013 et 15 novembre 2013-10 janvier 2014) et Miami (7 novembre-15 décembre 2013). Elle sera ouverte à Washington du 23 janvier au 23 mars. Ensuite elle sera exposée à Séoul.

33. El Bulli a fermé ses portes en 2011. Il rouvrira en 2015 comme Fondation consacrée à la recherche culinaire, à la formation de futurs chefs et à la diffusion nouvelles créations gastronomiques.

34. L'établissement Inopia a fermé en juillet 2010.

35. Pour avoir une place, il faut réserver au moins trois mois à l'avance, uniquement par internet. 36. Il s'agit du même local avec deux entrées différentes. Sur les nouveaux concepts de " $41^{\circ}$ » et «Tickets» on peut lire les articles de Marta Fernandez Guadaño, «41… ¿Se consolida la coctelería-snackería?», et "Tickets o el Bulli de barrio » http:// www.gastroeconomy.com/2011/07/el-caso-41\%C2\%BA-\%C2\%Bfllega-la-cocteleria-snackeria/; http://www.gastroeconomy.com/2011/07/tickets-o\%E2\%80\%A6-elbulli-de-barrio/, consulté le 15 février 2014.

37. Depuis la fermeture de El Bulli en 2011, Adrià a multiplié les interviews sur les chaînes de télévision et pour la presse spécialisée, les conférences sur la créativité et l'innovation dans le monde éducatif et de l'entreprise, notamment à Harvard en décembre 2011. En Juillet 2013 a eu lieu l'expo sur El Bulli dans le cadre de « Art of Food at Somerset House » à Londres.

\section{RÉSUMÉS}

Depuis sa première étoile Michelin comme chef de «El Bulli » (1990, Girona-Espagne), Ferran Adrià a poursuivi une longue carrière couronnée de succès, devenant ainsi la figure emblématique de la nouvelle cuisine espagnole. Sa démarche originale a transformé le "savoir faire » des chefs en une recherche personnelle alliant la créativité, la science et la technologie à une conception anthropologique de la cuisine au service de la personne et de la société. Même si «El Bulli », nommé à cinq reprises "meilleur restaurant du monde», est resté un restaurant d'élite, Adrià a réussi à se faire connaître du grand public et à lui transmettre son message à travers des entretiens télévisés, des documentaires, de la presse et d'autres moyens de communication. 


\section{AUTEUR}

\section{PALOMA OTAOLA}

Paloma OTAOLA est Professeur des Universités à la Faculté des Langues, Université Jean Moulin Lyon 3. Docteur en Philosophie (Universidad de Navarra, 1992) et en Musicologie (Université Catholique de Louvain, 1998). En tant qu'enseignante en Master LEA, elle aborde dans ses cours plusieurs sujets concernant l'Espagne actuelle, dont la nouvelle architecture, la nouvelle cuisine, la transition à la démocratie, etc. Par ailleurs, ses recherches en musicologie portent sur l'humanisme dans la pensée musicale au XVIe siècle, musique et critique musicale au XXe siècle et l'éclosion de la musique pop dans les années 60 . 